\title{
Subharmonic resonant excitation of edge waves by breaking surface waves
}

\author{
Nizar Abcha ${ }^{1}$, Tonglei Zhang ${ }^{1}$, Alexander Ezersky ${ }^{1}$, Efim Pelinovsky $^{2,3,4}$, and Ira Didenkulova ${ }^{2,5}$ \\ ${ }^{1}$ Morphodynamique Continentale et Côtière UMR6143, CNRS, Normandie Univ, UNICAEN, 14000 Caen, France \\ ${ }^{2}$ Nizhny Novgorod State Technical University n.a. R.E. Alekseev, 24 Minin Str., Nizhny Novgorod 603950, Russia \\ ${ }^{3}$ Institute of Applied Physics, 46 Uljanov Str., Nizhny Novgorod 603950, Russia \\ ${ }^{4}$ National Research University, Higher School of Economics, Nizhny Novgorod 603950, Russia \\ ${ }^{5}$ Marine Systems Institute at Tallinn University of Technology, Akadeemia tee 15A, 12618 Tallinn, Estonia \\ Correspondence to: Nizar Abcha (nizar.abcha@unicaen.fr)
}

Received: 24 October 2016 - Discussion started: 2 November 2016

Revised: 19 February 2017 - Accepted: 27 February 2017 - Published: 28 March 2017

\begin{abstract}
Parametric excitation of edge waves with a frequency 2 times less than the frequency of surface waves propagating perpendicular to the inclined bottom is investigated in laboratory experiments. The domain of instability on the plane of surface wave parameters (amplitude-frequency) is found. The subcritical instability is observed in the system of parametrically excited edge waves. It is shown that breaking of surface waves initiates turbulent effects and can suppress the parametric generation of edge waves.
\end{abstract}

\section{Introduction}

The study of parametric excitation of waves with half of their external frequency has a long history. The first papers on this subject were published by M. Faraday, who described excitation of capillary ripples with a frequency of $\Omega / 2$ in a thin horizontal layer of viscous fluid placed on a horizontal plate oscillating vertically with a frequency of $\Omega$ (Faraday, 1831). After Faraday, such parametric excitation of waves was observed in hydrodynamics (Douady, 1990; Cerda and Tirapegui, 1998), plasma physics (Okutani, 1967; Kato et al., 1965), chemically active media (Fermandez-Garcia et al., 2008), and other systems. Such parametric excitation also occurs in the ocean. Surface waves approaching the shore from the open sea with a frequency of $\Omega$ can excite the so-called edge waves with a frequency of $\Omega / 2$. Edge waves propagate along the coastline with their amplitudes decreasing in the offshore direction (Ursell, 1952; Grimshaw, 1974; Guza and
Davis, 1974; Evans and McIver, 1984; Johnson, 2005, 2007). Interest in parametrically excited edge waves is related to their ability to significantly affect morphological characteristics of sea coasts. Edge waves may contain enough energy to be responsible for beach erosion. They may also focus, forming a freak wave (Pelinovsky et al., 2010). Sometimes, edge waves are also associated with beach cusp formation (Guza and Imman, 1975; Komar, 1998; Masselink, 1999; Dodd et al., 2008; Coco and Murray, 2007).

Analytical solutions for edge waves excited by nonbreaking surface waves have been obtained in prior studies (Akylas, 1983; Minzoni and Whitham, 1977; Yeh, 1985; Yang, 1995; Blondeaux and Vittori, 1995; Galletta and Vittori, 2004; Dubinina et al., 2004). The correlation between characteristics of edge waves and spectra of surface waves approaching the shore is studied in situ (Huntley and Bowen, 1978). This kind of study is hard for analysis and interpretation of the results due to the irregularity of the coastline and the complex spectra of the approaching surface waves.

Laboratory experiments on parametric excitation of edge waves are described in Buchan and Pritchard (1995). The main advantage of such experiments is the freedom to define the bottom geometry and spectrum of the approaching surface waves. However, none of the studies mentioned above considered wave breaking, whereas in natural conditions surface waves often break while propagating towards the coastline. Thus, the influence of wave breaking on a parametric instability still remains an open question. In the present paper, 
we concentrate on the influence of wave breaking on characteristics of parametrically excited edge waves.

The paper is organized as follows. In Sect. 1, we focus on the theoretical description of the problem by providing the nonlinear equation for parametric excitation of edge waves. Section 2 is devoted to the experimental set-up, while Sect. 3 presents the results of measurements. In Sect. 4, we discuss the experimental data with respect to their theoretical interpretation. The main results are summarized in the conclusion.

\section{Theoretical model}

Let us start from the non-breaking scenario, when long waves propagate over some changing bottom geometry, $h=h(x)$. In this case, they can be described by 2-D nonlinear shallow water equations:

$$
\begin{aligned}
& \frac{\partial u}{\partial t}+u \frac{\partial u}{\partial x}+v \frac{\partial u}{\partial y}+g \frac{\partial \eta}{\partial x}=0 \\
& \frac{\partial u}{\partial t}+u \frac{\partial v}{\partial x}+v \frac{\partial v}{\partial y}+g \frac{\partial \eta}{\partial y}=0 \\
& \frac{\partial \eta}{\partial t}+\frac{\partial}{\partial x}(u(h+\eta))+\frac{\partial}{\partial y}(v(h+\eta))=0
\end{aligned}
$$

where $(u, v)$ are the two components of the depth-averaged horizontal velocity, $\eta=\eta(x, y, t)$ is the free surface displacement, and $g$ is the gravity acceleration. In a linear approximation, the system Eqs. (1)-(3) can be transformed into a 2-D wave equation:

$$
\frac{\partial^{2} \eta}{\partial t^{2}}-g\left(\frac{\partial}{\partial x}\left[h(x) \frac{\partial \eta}{\partial x}\right]+h(x) \frac{\partial^{2} \eta}{\partial y^{2}}\right)=0 .
$$

Note that Eq. (4) describes both surface waves propagating perpendicular to the shore and generated edge waves. For edge waves, we assume that they propagate along the shore and consider a linear change of the bottom slope $h(x)=$ $\beta x=\tan \alpha x$. In this case, an elementary solution of Eq. (4) has the following form:

$$
\begin{gathered}
\eta=b \cos \left(\Omega_{n} t-k y\right) \cdot e^{-k x} L_{n}(x), \\
\Omega_{n}=\sqrt{(2 n+1) \beta g k}, n=0,1,2, \ldots .
\end{gathered}
$$

where $L_{n}$ are the Laguerre polynomials, $b$ is a wave amplitude, $k$ is a wave number along the propagation direction, $\Omega$ is a wave frequency, and $n$ is the number of the mode.

By using two edge waves propagating in opposite directions, it is also possible to compose a solution corresponding to a standing edge wave:

$$
\begin{aligned}
\eta & =b \cos \left(\Omega_{n, m} t\right) \sin \left(k_{m} y\right) L_{n}(x), \\
k_{m} & =\pi(1+2 m) / L, \Omega_{n, m}=\sqrt{(2 n+1) \beta g k_{m}}, \\
m & =0,1,2, \ldots
\end{aligned}
$$

Here, we used the boundary conditions $v(x, y, t)=0$ at $y= \pm L / 2$, where $L$ is a channel width. For surface waves propagating perpendicular to the shore, Eq. (4) transforms into a 1-D wave equation,

$\frac{\partial^{2} \eta}{\partial t^{2}}-g \frac{\partial}{\partial x}\left(h(x) \frac{\partial \eta}{\partial x}\right)=0$,

and has a solution:

$\eta(x, t)=a_{0} J_{0}\left(\sqrt{\frac{4 \omega^{3} x}{g \beta}}\right) \cos (\omega t)$,

where $J_{0}$ is the Bessel function of the first kind, $\omega$ is a frequency, and $a_{0}$ is an amplitude of the generated surface waves.

In the linear approximation waves, Eqs. (6) and (8) are independent. If nonlinear effects are taken into consideration (Eqs. 1-3), coupling between the two types of waves takes place. In the first approximation of nonlinearity, surface waves described by Eq. (8) can generate edge waves described by Eq. (6) if $\Omega \approx \omega / 2$. It is the so-called parametric subharmonic resonance. In this case, we can write down the equation for slowly varying wave amplitude $b$ of the excited edge waves with frequency $\Omega$ (Rabinovich et al., 2000):

$\frac{\partial b}{\partial t}=-\gamma b+\mu b^{*}+i \Delta b+(i \sigma-\rho) b|b|^{2}$.

Here, $\gamma$ represents an exponential decay of edge waves due to the viscous dissipation, $\Delta=\Omega-\omega / 2$ is a detuning between frequencies of edge waves and the external parametric forcing, $\sigma$ is a nonlinear frequency shift, $\rho$ is a nonlinear damping coefficient, and $b^{*}$ is a complex conjugate. This equation was initially obtained for Faraday ripples excited by a homogeneous oscillating field. For edge waves excited by surface waves propagating perpendicular to the shore, an expression for a coefficient $\mu$ has been obtained in previous studies (Akylas, 1983; Minzoni and Whitham, 1977; Yang, 1995):

$\mu=a_{0} \frac{\omega^{3}}{4 g \beta^{2}} S(\beta)$.

Here, $S$ is a coefficient depending on a bottom slope $\alpha$. For small slopes $\alpha, S \approx 6.7 \times 10^{-2}$. The nonlinear frequency shift $\sigma$ has been calculated in Minzoni and Whitham (1977). The nonlinear damping coefficient $\rho$ has been discussed in Yang (1995).

\section{Experimental set-up}

The experiments have been performed in the wave flume of the Laboratory of Continental Coastal Morphodynamics of University of Caen Normandy, France. This flume has a length of $18 \mathrm{~m}$ and width of $0.5 \mathrm{~m}$. The flume is equipped with a piston type of wavemaker controlled by the computer. For construction of an inclined bottom slope, a PVC plate of 


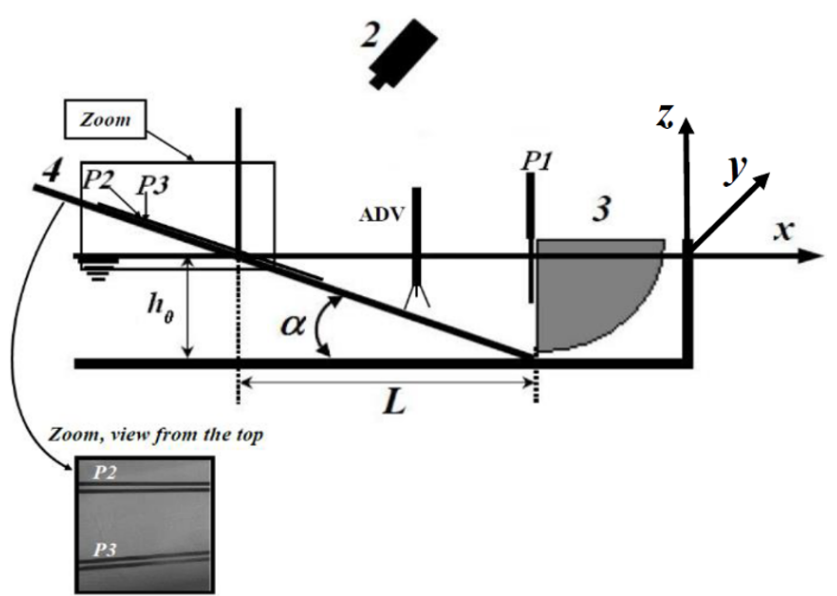

Figure 1. The experimental set-up: resistance probes: vertical (P1) and horizontal (P2, P3), a high-speed video camera (2), a wavemaker of a piston type (3), an inclined bottom (4), and the acoustic Doppler velocimeter (ADV).

$0.01 \mathrm{~m}$ thickness has been used. The plate has been placed at an angle $\alpha$ to the horizontal bottom so that $\tan \alpha=\beta=0.20$; the water depth in the flume, $h$, has been kept at $0.25 \mathrm{~m}$ (see Fig. 1). As one can see from Fig. 1, in this geometric configuration only a small part of the flume can be used for experiments. Three resistance probes - P1, P2, P3 (see Fig. 1) have been used to measure the water surface displacement.

The first of these, the immobile probe P1, has been placed at a distance of $1 \mathrm{~cm}$ from the wavemaker, while probes $\mathrm{P} 2$ and P3 have been glued to the inclined plate. The latter two probes placed along the bottom slope allow us to measure wave run-up and run-down. In addition, the run-up height can be identified by image processing from the high-speed camera operating with a frame rate of $100 \mathrm{~Hz}$ (see Fig. 1). The wavemaker oscillating with a given frequency and amplitude allows us to excite the targeted mode described by Eq. (8). The wavemaker can work in two regimes. The first regime controls the amplitude of the wavemaker displacement, while the second one controls the amplitude of the force applied to the wavemaker. In both regimes, it is not possible to control the free surface displacement. Therefore, to study the surface wave characteristics, simultaneous measurements of a free surface displacement near the wavemaker and the shoreline have been carried out. For velocity fields (all three components of the flow velocity), the acoustic Doppler velocimeter (ADV) has been used. The quality of the signal registered by ADV strongly depends on the concentration of particles in the liquid. Therefore, in order to get a better signal, some small particles with a diameter of $10 \mu \mathrm{m}$ have been added into the water.

For visualization of a free surface displacement in the breaking zone by the high-speed camera, the water has also been seeded with sand particles of $10 \mu \mathrm{m}$. Using a vertical light sheet (photodiode $532 \mathrm{~nm}$ with a cylindrical lens), it

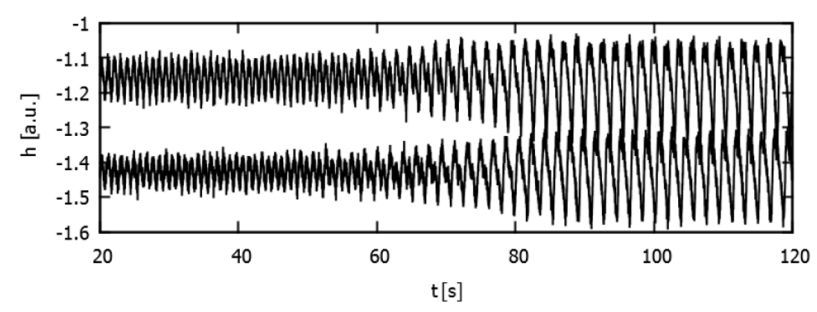

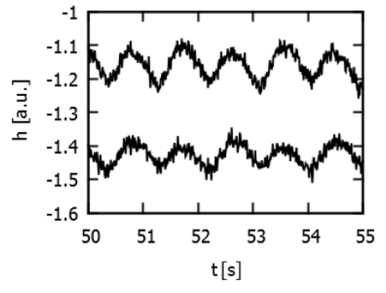

(b) (a)

Figure 2. Example of wave instability developing from a natural perturbation with $f=1.08 \mathrm{~Hz}, a_{L}=0.66 \mathrm{~cm}$ : (a) the full time series recorded by probes $\mathrm{P} 2$ and $\mathrm{P} 3$; (b) zoom of the time series recorded during the time interval $50 \mathrm{~s}<t<55 \mathrm{~s}$, and (c) during the time interval $85 \mathrm{~s}<t<90 \mathrm{~s}$.

has been possible to visualize the cross section of the water in the $x-z$ plane. The size of the visualization domain is $40 \mathrm{~cm} \times 30 \mathrm{~cm}$.

Our excitation frequency range was chosen following our published study about the physical simulation of resonant wave run-up on a beach (see Ezersky et al., 2013). In this study, we describe edge waves excited by the third resonant mode of the system.

\section{Data processing and results}

The subharmonic instability described above is investigated in the flume for different values of $\left(a_{L}, f\right)$, where $a_{L}$ is an amplitude of surface waves in the vicinity of the wavemaker, $a_{L} \approx a_{0}$, and $f$ is the frequency of the wavemaker. In order to understand whether the instability really occurs, we analyse the signals from probes P2 and P3. Before each experiment, we wait for 5-10 min to let all the perturbation in the flume decay and let the wavemaker work in calm water conditions.

An example of the signals from P2 and P3 is shown in Fig. 2a, whereas a more detailed zoom of the time series for intervals $50 \mathrm{~s}<t<95 \mathrm{~s}$ and $85 \mathrm{~s}<t<90 \mathrm{~s}$ is given in Fig. $2 \mathrm{~b}$ and c, respectively. The power frequency spectra for two surface wave regimes (with and without wave breaking) are shown in Fig. 3. The first spectrum (Fig. 3a) is the fast Fourier transform (FFT) of the signal shown in Fig. 2a. This is a spectrum in the absence of wave breaking, where the first peak indicates the edge wave frequency and the second peak indicates the surface elevation frequency. The second frequency spectrum (Fig. 3b) is plotted in the presence of 


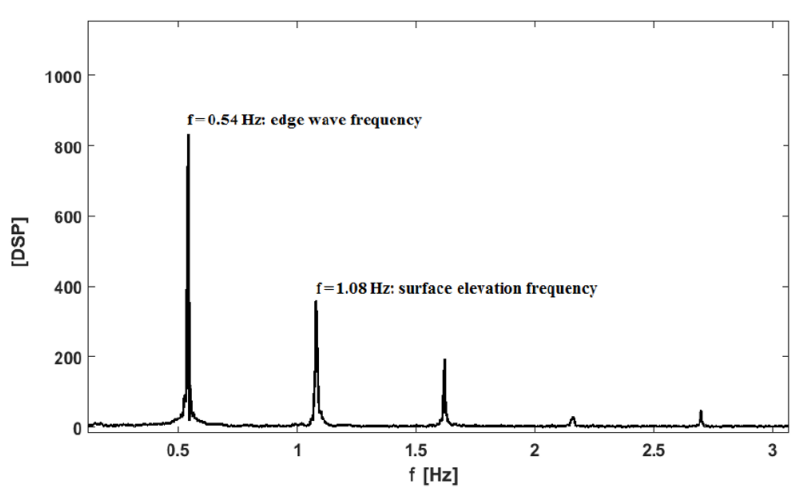

(a)

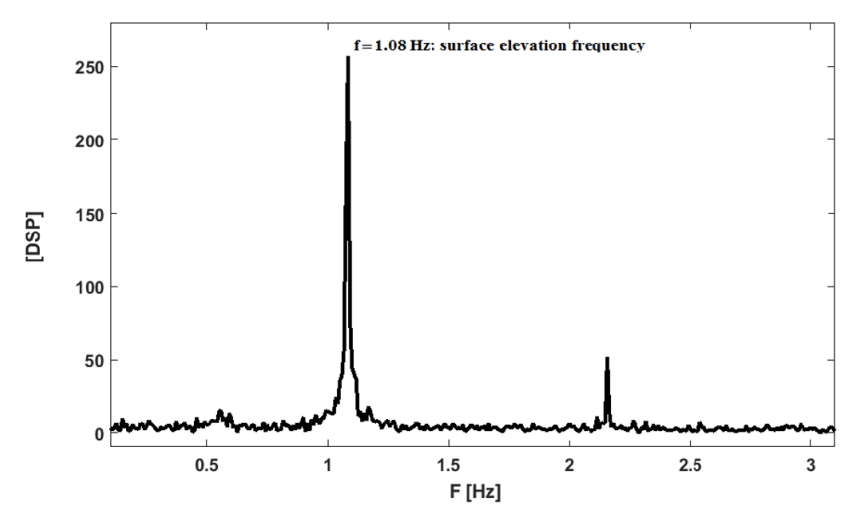

(b)

Figure 3. Power spectrum frequency: (a) in the absence of breaking waves, the first peak indicates the edge wave frequency, while the second peak indicates the surface elevation frequency; (b) in the presence of breaking waves, the peak for the edge wave frequency is suppressed.

breaking waves and indicates the suppression of the peak for the edge wave frequency.

It can be seen that in the beginning of the record the waves have the same frequency and phase as the wavemaker (Fig. 2b). However, after instability arises (Fig. 2c), the amplitude of generated edge wave increases and the period doubles compared to the period of surface waves. The phase shift between the signals recorded by probes $\mathrm{P} 2$ and $\mathrm{P} 3$ is approximately $\pi$. These two criteria (period doubling and a phase shift equal to $\pi$ ) are used to identify parametric instability. To confirm an appearance of edge waves as a result of subharmonic instability, we analyse the water level oscillations. It is found that subharmonic oscillations represent the mode, where maxima of horizontal displacement (antinodes) occur near the lateral walls of the flume, while its zeroes (nodes) are observed in the middle of the flume. This mode is a superposition of two edge waves propagating in opposite directions. A spatial period of these edge waves is 2 times larger than the width of the flume. Snapshots of water surface over the time interval equal to half of the edge wave period are shown in Fig. 4.
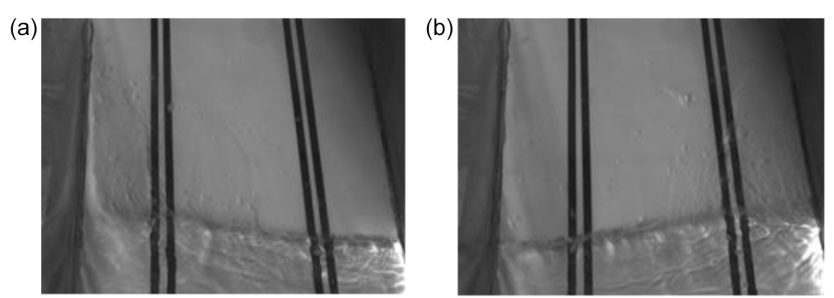

Figure 4. Snapshots of water surface over the time interval equal to half of the edge wave period (approximately $1 \mathrm{~s}$ ), $f=1.06 \mathrm{~Hz}$, and $a_{L}=1.3 \mathrm{~cm}$.

Subharmonic instability starts with an exponential growth of an infinitely small perturbation. To describe the instability in the system, partitioning of a $\left(a_{L}, f\right)$ plane into different stability regions is performed. Results of this analysis are demonstrated in Fig. 5.

Instability occurs if the frequency of surface waves is close to a double frequency of edge waves. Curve 1 represents a border of the supercritical instability regime which occurs for points $\left(a_{L}, f\right)$ above this curve. If the amplitude of surface waves decreases from a finite value above Curve 1, generation of edge waves is observed in a small region (3) between Curves 1 and 2 (see triangles in Fig. 5). When we start from the regime without edge wave generation (points below Curve 2) and increase the amplitude of surface waves, instability will occur above Curve 1 . This type of instability is called subcritical instability.

The partition of a plane $\left(a_{L}, f\right)$ into regions with different regimes shown in Fig. 5 corresponds to two qualitatively different conditions of wave excitation schematically shown by boxes (I) and (II). In Region I, surface waves excited by the wavemaker and propagating to the shore undergo a plunging wave breaking. In Region II, waves do not break. Image processing of the high-speed camera data shows that such excitation occurs only when the wave breaking parameter $\mathrm{Br}>0.9$. Under the wave breaking parameter, we mean $\mathrm{Br}=U_{\max }^{2} / \mathrm{gR}$, where $U_{\max }$ is the maximal flow velocity and $R$ is the maximal wave run-up height on the shore (Didenkulova, 2009).

It is found that while surface wave breaking leads to the appearance of the hydrodynamic turbulence, turbulence itself leads to a decrease in the amplitude of excited edge waves and suppression of subharmonic generation for largeamplitude surface waves.

Dependences of the increment of edge wave instability and intensity of turbulent velocity fluctuation on the amplitude of surface waves $a_{L}$ are shown in Fig. 6a and b. The dependence of the exponential index $\gamma$ on the amplitude of surface waves $a_{L}$ is found by processing corresponding time series similar to those shown in Fig. 2a. For this, we select time intervals where the edge wave amplitude grows and calculate $\gamma$ by exponential approximation of the time-dependent amplitude. 


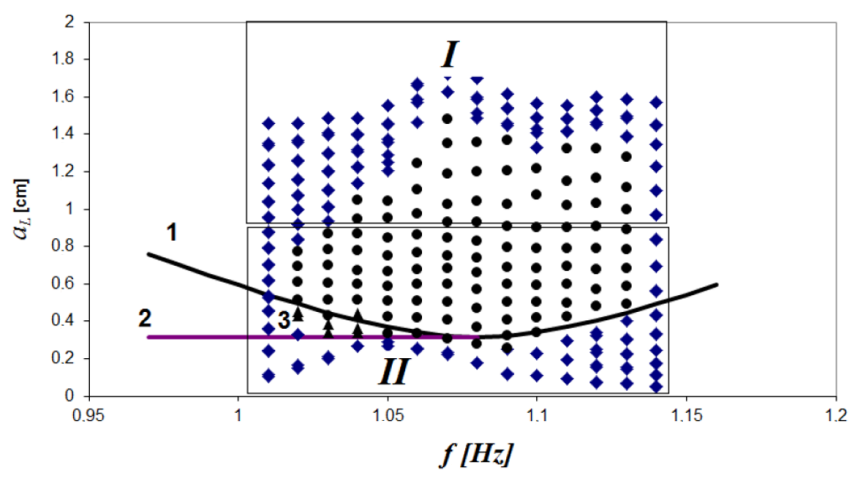

Figure 5. Partition of a $\left(a_{L}, f\right)$ plane into different stability regions of the system; circles correspond to a parametric instability, diamonds correspond to stability regimes, and triangles indicate the regime of subcritical instability.

Parameters of the turbulence are measured by ADV in the middle of the experimental flume, $0.04 \mathrm{~m}$ below the free surface $(0.14 \mathrm{~m}$ from the bottom), at a distance of $x=0.9 \mathrm{~m}$ from the shoreline. At this point, it is possible to neglect the turbulence caused by the near-bottom oscillating boundary layer and detect the wave breaking turbulence.

Here, we should specify some difficulties related to the characteristic features of ADV signals. The recorded ADV signals contain the so-called spikes, which are filtered using the MATLAB algorithm (Nikora and Goring, 1998; Goring and Nikora, 2002). Another problem occurs due to the complex structure of the velocity field in the breaking zone, which represents a mixture of turbulence and velocities caused by both surface and edge waves. In this case, the impact of surface and edge wave components is removed by filtering harmonics with frequencies $f / 2, f, 3 f / 2,2 f, 5 f / 2$, and $3 f$. It is shown that the intensity of turbulence grows sufficiently if the amplitude of surface waves $a_{L}$ is larger than $0.8 \mathrm{~cm}$ (see Fig. 6b).

\section{Discussion}

Thus, the range of parameters corresponding to the parametric excitation of edge waves is found experimentally. Now, using the theoretical Eq. (10), we can estimate the threshold of parametric excitation of edge waves. For this, we need to find the eigenfrequencies of edge waves in the flume $\Omega_{n}$. The frequency of the zero edge wave mode $\Omega_{0}$ is as follows:

$\Omega_{0}=\sqrt{\beta g \frac{\pi}{L}}=3.41 \mathrm{rad} \mathrm{s}^{-1}, f_{0} \approx 0.54 \mathrm{~Hz}$.

To estimate the dissipation rate of edge waves, we study the time evolution of the edge wave amplitude after stopping the parametric excitation. Edge waves decay exponentially and in this way we measure the decay rate $\gamma$, which is estimated as $\gamma=0.1 \mathrm{~s}^{-1}$. For the resonance condition $\Delta=0$, parametric instability occurs when the wave amplitude exceeds the critical wave amplitude $a_{0}$ :

$a_{0}=\gamma \frac{4 g \beta^{2}}{\omega^{3} S(\beta)} \approx 0.76 \mathrm{~cm}$

The theoretical value of the parametric instability threshold is calculated using the free surface displacement. To compare experimental and theoretical values of the threshold, we need to measure the surface wave amplitude at $x=0$. As it has been noted in several studies (see, for example, Denissenko et al., 2011), this value can be measured indirectly. We find it using the visualization of the flow in the middle of the flume by the laser sheet at a time preceding the development of the edge wave parametric instability (see Fig. 7).

Note that while the parametric instability threshold is determined, there was no surface wave breaking, which corresponds to Region II in Fig. 5.

Figure 7 shows what occurs before the development of the parametric instability, when amplitudes of edge waves are zero. To estimate the surface wave amplitude, the measured crest-to-trough wave height (Fig. 7) is divided by 2. Comparison of the experimental and theoretical values of the instability threshold is shown in Fig. 8. One can see from Fig. 8 that theoretical values are larger than experimental ones by approximately $30 \%$.

Note that even when the surface wave breaking takes place, the parametric excitation of edge waves still occurs. However, the parametric excitation is suppressed for large amplitudes of surface waves. The reason for this could be the following. The wave breaking results in the irregularity of the surface wave field: amplitudes and phases of the waves vary chaotically. Evidently, wave breaking also leads to the appearance of small-scale turbulence in the near-shore zone. Below, we discuss the impact of these two physical mechanisms on the suppression of the parametric instability.

The parametric wave excitation by the irregular oscillating field has been studied in Ezersky and Matusov (1994) and Nikora et al. (2005). It was shown that chaotic amplitudes and phases of the external wave field lead to an increase in the threshold of parametric excitation and decrease in the amplitude of parametrically excited oscillations.

Let us check whether these results can explain the decrease in the edge wave amplitude in the presence of the wave breaking. For this, we calculate amplitudes and phases of surface waves. After narrow-band filtering generated by the wavemaker, surface waves may be described as $\eta_{m} \cos (\omega t+$ $\Phi)$, where $\eta_{m}$ is a slow varying amplitude and $\Phi$ is a slow varying phase. To extract the amplitude and the phase of the signal, the Hilbert transformation is used:

$\hat{\eta}(t)=\frac{1}{\pi} \mathrm{PV}\left[\int_{-\infty}^{+\infty} \frac{\eta(t, \tau)}{t-\tau} \mathrm{d} \tau\right]=\eta_{m} \sin (\omega t+\Phi)$, 


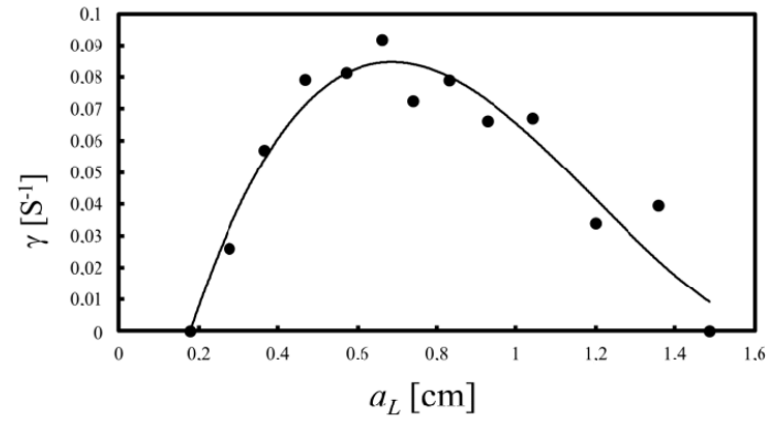

(a)

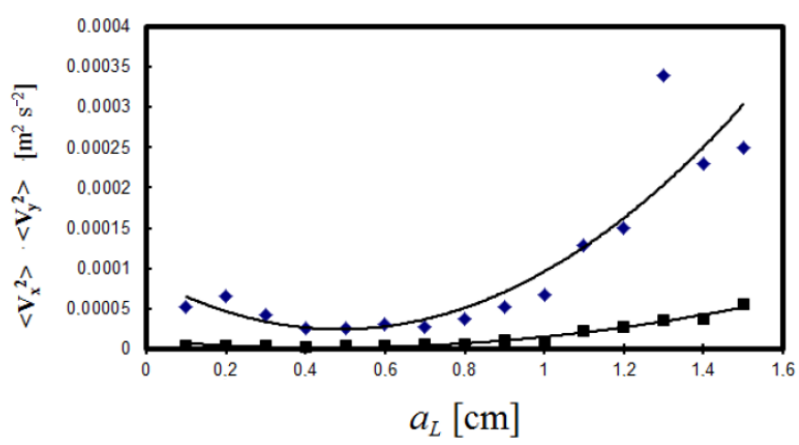

(b)

Figure 6. (a) Dependence of the exponential index of parametric instability $\gamma$ on the surface wave amplitude $a_{L}$, shown by the black dots, and (b) dependence of the kinetic turbulent energy components on the surface wave amplitude $a_{L} ; V_{x}$ is shown by blue diamonds, while $V_{y}$ is shown by black squares. Solid lines represent a fit to the experimental data.

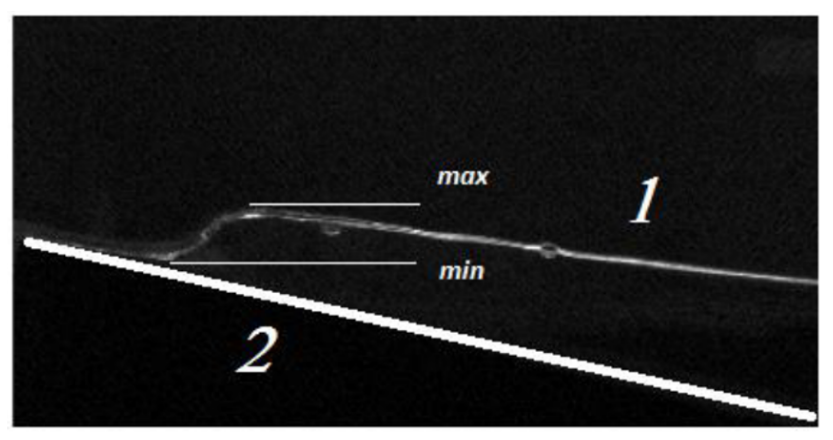

Figure 7. Visualization of the free surface displacement: 1 indicates the water surface; 2 indicates the inclined bottom; max and min correspond to the maximum and minimum values of the free surface displacement.

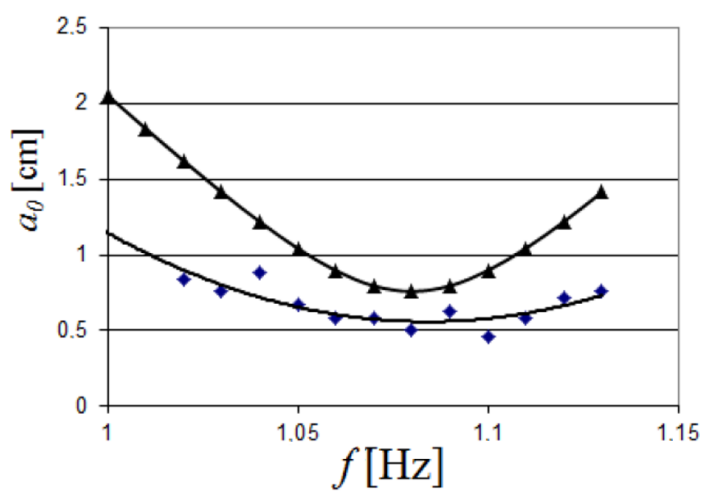

Figure 8. Comparison of experimental and theoretical values of the instability threshold: triangles correspond to the theoretical formula; diamonds represent experimental data.

where PV denotes the principal value of the integral. It is also possible to determine the wave amplitude and phase:

$\eta(t)=\operatorname{Re}\left\{a(t) \exp (i \omega t\}, a(t)=|a| e^{i \Phi}\right.$, where

$|a|=\sqrt{\eta^{2}+\hat{\eta}^{2}}, \Phi=\arctan (\hat{\eta} / \eta)-\omega t$.

Extracted amplitudes and phases for the time series measured in the presence of the surface wave breaking are shown in Fig. 9. The time series itself is given in Fig. 9a, while the extracted amplitudes and phases are shown in Fig. 9b. The root mean square of phase and amplitude fluctuations for the intensive wave breaking $(a=1.4 \mathrm{~cm})$ is

$\sqrt{\left\langle\Phi^{2}\right\rangle} \approx 0.1, \frac{\sqrt{\left\langle(a-\langle a\rangle)^{2}\right\rangle}}{\langle a\rangle} \approx 0.1$.

It is also possible to estimate the influence of chaotic phases and amplitudes on the parametric wave excitation. It has been revealed that chaotic phases decrease the effective amplitude of the external force (Petrelis et al., 2005). Suppose that the wave breaking leads to the Gaussian noise; then, the corresponding decrease in the external forcing may be estimated as (Petrelis et al., 2005)

$e^{-\left(\left\langle\Phi^{2}\right\rangle / 2\right.} \approx 0.995$.

This small decrease in the effective external forcing cannot explain suppression of the parametric excitation during the wave breaking regime; therefore, the influence of the turbulence seems to be more important.

Wave breaking generates turbulence, and the intensity of turbulent velocity fluctuations grows with the surface wave amplitude. On the other hand, turbulence leads to the appearance of turbulent shear stresses and eddy viscosity $v_{\text {ed }}$. We measure experimentally some components of the kinematic turbulent energy at the edge wave background (see Fig. 6b). According to our measurements, the most important components of shear stresses are related to the longitudinal component of turbulent fluctuations $V_{x}$ (see Fig. 6b).

The eddy viscosity $\nu_{\text {ed }}$ is proportional to the turbulent energy. For the wave breaking case, one can consider $v_{\text {ed }}$ to be 


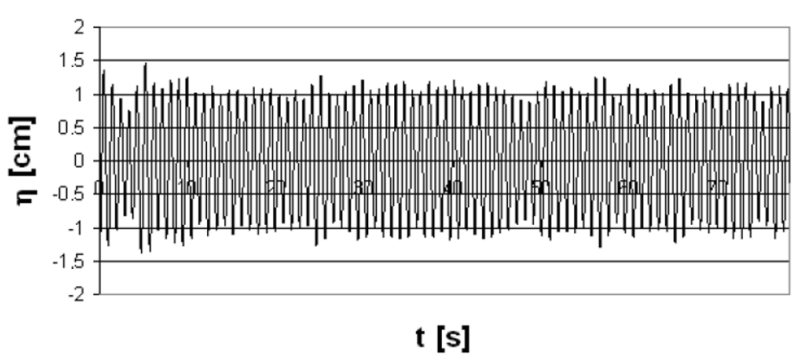

(a)

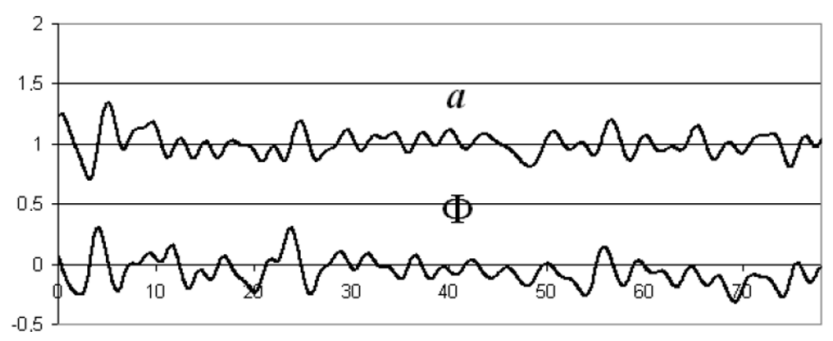

t [s]

(b)

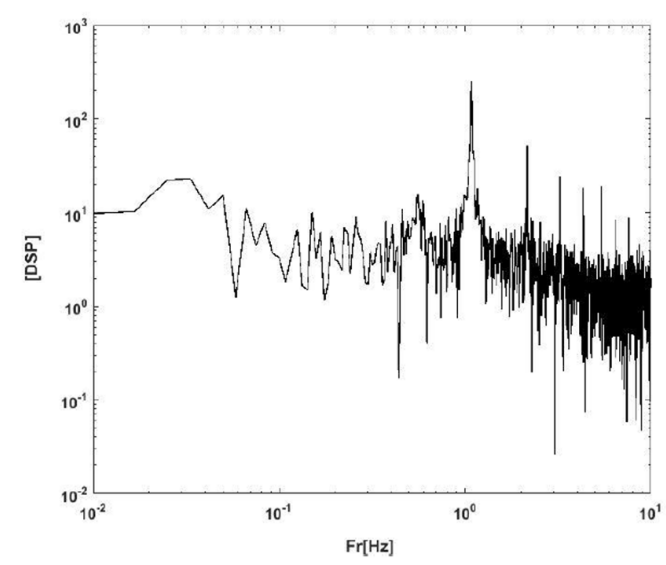

(c)

Figure 9. (a) Time series measured by $\mathrm{P} 1$ with $a_{L}=1 \mathrm{~cm}$, $f=1.06 \mathrm{~Hz}$; (b) non-dimensional wave amplitude and phase obtained by the Hilbert transformation; (c) power spectrum of the signal (a) in $\log -\log$ scale.

proportional to $a^{2}$ (see Fig. 6b). In this case, the exponential decay $\gamma$ in Eq. (9) has the following form: $\gamma=\gamma_{0}+\gamma_{1} a^{2}$, where $\gamma_{0}$ is the exponential decay of edge waves in the absence of wave breaking, and $\gamma_{1}$ is responsible for energy dissipation due to the eddy viscosity.

Since the external forcing $\mu$ grows linearly with the surface wave amplitude and the dissipation is proportional to the amplitude squared, the parametric instability is suppressed for large surface wave amplitudes. We observe this effect in our experiment under the surface wave breaking regime.

From the measurements, to calculate the energy dissipation rate, we have study the energy of wave propagating in the

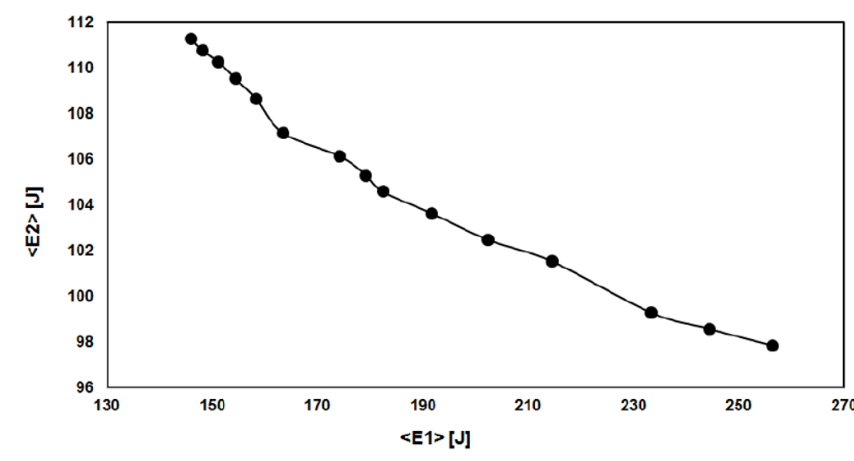

Figure 10. Dependence of wave-propagating energy $(f=1.06 \mathrm{~Hz})$ E2 (energy at shore) on E1 (energy near the wavemaker) for different amplitudes of excitation $a_{L}$.

flume. We compare wave energy near the wavemaker with wave energy at shore. The wave energy (energy on a unit length in the direction transversal to the direction of wave propagation) is estimated as follows:

$E=\frac{\rho g}{2} C_{\mathrm{gr}} \int(\eta-<\bar{\eta}>)^{2} \mathrm{~d} t$,

where $C_{\mathrm{gr}}=\frac{\mathrm{d} \omega}{\mathrm{d} K}$ is the group velocity of the harmonic component corresponding to the peak frequency $f ; g$ is acceleration of gravity; $\rho$ is water density; and $\eta$ and $<\bar{\eta}>$ are free surface displacement and mean water level, respectively.

The typical dependence of E2 (energy at shore) on E1 (energy near the wavemaker) is shown in Fig. 10 for different amplitudes of excitation for $f=1.06 \mathrm{~Hz}$. We observe that evolution of dependence follows a law of power. An energy dissipation of the order of $25 \%$ occurs in the absence of the edge waves. These losses are caused by viscous dissipation and contact line damping. In the presence of edge waves, the energy dissipation can reach $50 \%$.

\section{Conclusions}

The parametric edge wave excitation is studied for different regimes of surface wave propagation. We have found that for parametrically excited edge waves there is a region of subcritical instability, which is manifested by the hysteresis: different regimes of edge wave excitation are observed in the case of decrease or increase in the surface wave amplitude. Note that subcritical instability was not observed in Buchan and Pritchard (1995), though their experimental conditions were very close to those in our experiment.

The increase in the surface wave amplitude leads to the appearance of wave breaking. The wave breaking regime itself does not prevent parametric excitation of edge waves; only the developed wave breaking can suppress parametric excitation of edge waves. We compare the two possible mechanisms of the parametric instability suppression: (i) phase irregularity of the external forcing and (ii) generation of the 
hydrodynamic turbulence. We have found that the most probable mechanism responsible for the increase of the parametric instability threshold and suppression of parametric excitation of edge waves is the hydrodynamic turbulence which appears as a result of wave breaking.

Data availability. The data used by this study are experimental. The data are freely available but not otherwise published in any publicly accessible database. The experimental data can nonetheless be provided upon request via email to the corresponding author, Nizar Abcha (nizar.abcha@unicaen.fr).

Competing interests. The authors declare that they have no conflict of interest.

Acknowledgements. This work is dedicated to Alexander Ezersky, who was the key author and the main driver of this study. Last summer, he sadly passed away after a long-lasting fight with cancer, leaving the manuscript unfinished. Until his last days, he tried to dedicate his time to work, including the results presented here. Therefore, it is important for us to conclude his work in memory of a dear friend and colleague.

The present study was supported by Russian Presidential grants MD-6373.2016.5 and NS-6637.2016.5. Ira Didenkulova and Efim Pelinovsky also thank the visitor programme of the University of Caen Normandy, which allowed this fruitful collaboration.

Edited by: R. Grimshaw

Reviewed by: two anonymous referees

\section{References}

Akylas, T. R.: Large-scale modulations of edge waves, J. Fluid Mech., 132, 197-208, 1983.

Blondeaux, P. and Vittori, G.: The nonlinear excitation of synchronous edge waves by a monochromatic wave normally approaching a plane beach, J. Fluid Mech., 301, 251-268, 1995.

Buchan, S. J. and Pritchard, W. G.: Experimental observations of edge waves, J. Fluid Mech., 288, 1-35, 1995.

Cerda, E. A. and Tirapegui, E. L.: Faraday's instability in viscous fluid, J. Fluid Mech., 368, 195-228, 1998.

Coco, G. and Murray, B. A.: Pattern in the sand: form forcing templates to self-organization, Geomorphology, 91, 271-290, 2007.

Denissenko, P., Didenkulova, I., Pelinovsky, E., and Pearson, J.: Influence of the nonlinearity on statistical characteristics of long wave runup, Nonlin. Processes Geophys., 18, 967-975, doi:10.5194/npg-18-967-2011, 2011.

Didenkulova, I.: New trends in the analytical theory of long sea wave runup, in: Applied Wave Mathematics: Selected Topics in Solids, Fluids, and Mathematical Methods, Springer, 265-296, 2009.

Dodd, N., Stoker, A. M., Calvete, D., and Sriariyawat,A.: On beach cusp formation, J. Fluid Mech., 597, 145-169, 2008.
Douady, S.: Experimental study of the Faraday instability, J. Fluid Mech., 221, 383-409, 1990.

Dubinina, V. A., Kurkin, A. A., Pelinovsky, E. N., and Poloukhina, O. E.: Weakly nonlinear periodic Stokes edge waves, Izvestiya, Atmos. Ocean. Phys., 40, 464-469, 2004.

Evans, D. V. and McIver, P.: Edge waves over a shelf: full linear theory, J. Fluid Mech., 142, 79-95, 1984.

Ezersky, A. B. and Matusov, P. A.: Time-space chaos of capillary waves parametrically excited by noise, Radiophys. Quantum El., 37, 828-836, 1994.

Ezersky, A., Abcha, N., and Pelinovsky, E.: Physical simulation of resonant wave run-up on a beach, Nonlin. Processes Geophys., 20, 35-40, doi:10.5194/npg-20-35-2013, 2013.

Faraday, M.: On the forms and states of fluids on vibrating elastic surfaces, Philos. T. R. Soc. Lond. 52, 319-340, 1831.

Fernández-García G., Roncaglia D. I., Pérez-Villar V. Muñuzuri A. P, and Pérez-Muñuzuri V.: Chemical-wave dynamics in a vertically oscillating fluid layer, Phys. Rev. E, 7, 026204, doi:10.1103/PhysRevE.77.026204, 2008.

Galletta, V., and Vittori, G.: Nonlinear effects on edge wave development, Eur. J. Mech. B-Fluid. 23, 861-878, 2004.

Goring, D. and Nikora, V.: Despiking acoustic Doppler velocimeter, J. Hydraul. Eng., 128, 117-126, 2002.

Grimshaw, R.: Edge waves: a long wave theory for oceans of finite depth, J. Fluid Mech., 62, 775-791, 1974.

Guza, R. T. and Davis, R. E.: Excitation of edge waves bywaves incident on a beach, J. Geophys. Res., 79, 1285-1291, 1974.

Guza, R. T. and Inman, D. L.: Edge waves and beach cusps, J. Geophys. Res., 80, 2997-3012, 1975.

Huntley, D. A. and Bowen, A. J.: Beach cusps and edge waves, Proc 16th Conf. Coastal, 1378-1393, 1980.

Johnson, R. S.: Some contributions to the theory of edge waves, J. Fluid Mech., 524, 81-97, 2005

Johnson, R. S.: Edge waves: theories past and present, Philos. T. R. Soc. A, 365, 2359-2376, 2007.

Kato, K., Yoseli, M., and Kiyama, S.: Excitation of plasma oscillation by parametric resonance, J. Phys. Soc. Jpn., 20, 2097-2098, 1965.

Komar, P.: Beach Processes and Sedimentation, Prentice Hall, New York, 1998.

Masselink, G.: Alongshore variation in beach cusp morphology in a coastal embayment, Earth Surf. Proc. Land., 24, 335-347, 1999.

Minzoni, A. A. and Whitham, G. B.: On the excitation of edge waves on beaches, J. Fluid Mech., 79, 273-287, 1977.

Nikora, V. and Goring, D.: ADV measurements of turbulence: Can we improve their interpretation?, J. Hydraul. Eng., 124, 630-634, 1998.

Okutani, J.: Excitation of plasma oscillations by parametric resonance, J. Phys. Soc. Jpn., 23, 110-113, 1967.

Pelinovsky, E., Polukhina, O., and Kurkin, A.: Rogue edge waves in the ocean, Eur. Phys. J.-Spec. Top., 185, 35-44, 2010.

Petrelis, F., Aumattre, S., and Fauve, S.: Effect of Phase Noise on Parametric Instabilities, Phys. Rev. Lett. 94, 070603, doi:10.1103/PhysRevLett.94.070603, 2005.

Rabinovich, M. I., Ezersky, A. B., and Weidman, P. D.: The Dynamics of Pattern, World Sci., Singapore, 2000.

Ursell, F.: Edge waves on a sloping beach, P. R. Soc. A, 214, 79-97, 1952. 
Yang, J.: The stability and nonlinear evolution of edge wave, Stud. Appl. Math., 95, 229-246, 1995.
Yeh, H.: Nonlinear progressive edge waves: their instability and evolution, J. Fluid Mech. 152, 479-499, 1985. 